\title{
CA19-9 as a predictor of tumor response and survival in patients with advanced pancreatic cancer treated with gemcitabine based chemotherapy
}

\author{
Nazik HAMMAD, ${ }^{1}$ Lance K HEILBRUN, ${ }^{2}$ Philip A PHILIP, ${ }^{1}$ Anthony F SHIELDS, ${ }^{1}$ \\ Mark M ZALUPSKI, ${ }^{3}$ Raghu VENKATRAMANAMOORTHY ${ }^{2}$ and Bassel F EL-RAYES ${ }^{1}$ \\ Division of Hematology and Oncology, ${ }^{1}$ Karmanos Cancer Institute, Wayne State University, Detroit, ${ }^{3}$ University of Michigan \\ Cancer Center, Ann Arbor, MI and ${ }^{2}$ Biostatistics Unit, Karmanos Cancer Institute, Detroit, MI, USA
}

\begin{abstract}
Aims: The aim of this study was to determine the predictive role of pretreatment carbohydrate antigen 19-9 (CA19-9) measurement and its change after one cycle of gemcitabine-based therapy for response, time to progression (TTP) and overall survival (OS).

Methods: Analyses were derived from three consecutive gemcitabine-containing phase II clinical trials between 1997 and 2004.

Results: A total of 111 patients with pancreas cancer was studied. Baseline CA19-9 concentrations were dichotomized near the median. Lower baseline CA19-9 levels were positively associated with OS (median 9.1 vs 6.1 months, $P=0.0057$ ) and TTP (median 6.4 vs 4.2 months, $P=0.0044$ ). The covariate adjusted hazard ratio (HR) for progression among patients with baseline CA19-9 $\geq 1000 \mathrm{ng} / \mathrm{mL}$ was $\mathrm{HR}=1.94$ (95\% CI 1.24-3.02), with $P=0.0035$. The covariate adjusted risk of death among patients with baseline CA19-9 $\geq 1000 \mathrm{ng} / \mathrm{ml}$ was similarly elevated: $\mathrm{HR}=1.90$ (95\% CI $1.23-2.94)$, with $P=0.0039$. Change in CA19-9 levels from baseline to the end of treatment cycle 1 did not predict objective response $(P=0.75)$. There was somewhat longer OS (median $8.7 v s 7.1$ months) and TTP (median 7.1 vs 5.4 months) in patients with $\geq 50 \%$ reduction in serum CA19-9 concentrations, but this was not statistically significant $(P=0.74$ and 0.81 , respectively).

Conclusion: Baseline CA19-9 levels may predict survival in patients with advanced pancreas cancer. The change in CA19-9 levels determined within 1 month of the initiation of therapy did not predict treatment outcome.
\end{abstract}

Key words: CA19-9, gemcitabine, pancreatic cancer.

\section{INTRODUCTION}

Pancreatic cancer is the fourth leading cause of cancer death in the USA. The 5-year survival rate is generally less than $5 \% .{ }^{1}$ The mainstay of treatment of locally advanced and metastatic pancreatic cancer is chemotherapy. Gemcitabine, the most active chemotherapeutic

Correspondence: Dr Bassel F. El-Rayes MD, Division of Hematology and Oncology, Emory University, 1365 Clifton Road NE, Atlanta, GA 30332, USA.

Email: bassel.el-rayes@emoryhealthcare.org

Accepted for publication 28 February 2010. agent in pancreatic cancer, has shown a clinical benefit response rate of $24 \%$ and a survival rate at 1 year of $18 \% .^{2}$ A major challenge in the optimum delivery of therapy to patients with pancreatic cancer is the objective evaluation of tumor response, including the lack of predictive biomarkers. ${ }^{3}$ Response evaluation by crosssectional imaging is limited by the anatomic location of the pancreas and the marked fibrosis that surrounds tumor cells. Efforts are underway to develop alternative methods of evaluating response to guide earlier treatment decisions. This would help to avoid futile therapy and improve the risk stratification of patients in clinical trials. 
Table 1 Description of phase II clinical trials used in the pooled analysis of carbohydrate antigen 19-9 (CA19-9) measurements and patient outcome

\begin{tabular}{llccc}
\hline Study description & \multicolumn{1}{c}{ Period } & $\begin{array}{c}\text { No. of } \\
\text { patients in } \\
\text { the study }\end{array}$ & $\begin{array}{c}\text { Patients } \\
\text { with baseline } \\
\text { CA19-9 value }\end{array}$ & $\begin{array}{c}\text { Patients with CA19-9 } \\
\text { at baseline and after one } \\
\text { cycle of chemotherapy }\end{array}$ \\
\hline Gemcitabine and cisplatin $^{20}$ & May 1997 to March 1999 & 42 & 40 & 30 \\
Gemcitabine, cisplatin and 5-FU $^{21}$ & April 1999 to November 2001 & 47 & 43 & 27 \\
Gemcitabine, celecoxib and cisplatin & January 2002 to May 2003 & 22 & 19 & 11 \\
Total & Jan & 111 & 102 & 68 \\
\hline
\end{tabular}

The carbohydrate antigen 19-9 (CA19-9) is the most widely used serum tumor marker in pancreatic cancer, and was first described in $1979 .{ }^{4}$ The epitope of this antibody was subsequently identified as a sialylated lacto- $N$-fucopentaose II related to the Lewis a blood group antigen. Approximately $5 \%$ of the general population is genotypically Lewis ${ }^{\mathrm{a}-\mathrm{b}}$ and do not express CA19-9 because of an inherited deficiency of a fucosyltransferase specified by the Le gene. ${ }^{5}$ Approximately $15-20 \%$ of pancreatic cancer patients will not have an elevated CA19-9 at baseline. Pre-operative CA19-9 levels have been shown to predict recurrence and survival in patients with resected pancreatic cancer but at the time of writing do not influence the decision to operate. ${ }^{6}$

Serial measurements of serum CA19-9 levels in patients undergoing systemic therapy are used as adjuncts for evaluating response. However, it is not known what magnitude of change from the baseline level might be associated with radiological tumor response. Moreover, an early measurement of CA19-9 after one cycle of therapy may be a surrogate marker for a subsequent radiological response, which is usually evaluated after the second or third cycle of therapy. The aim of this study was to determine the role of baseline CA19-9 measurements and their change following the first cycle of gemcitabine-based therapy as potential predictors of tumor response, time to progression (TTP), and overall survival (OS).

\section{METHODS}

\section{Patients}

This study was an analysis of prospectively collected data pooled from three phase II studies examining gemcitabine and cisplatin alone or in combination with other agents. All three studies were undertaken at the Karmanos Cancer Institute in Detroit and the University of Michigan in Ann Arbor. The three trials are described in Table 1 and are all published. Patients were previously untreated with gemcitabine or any other chemotherapy except for their advanced disease.

To be eligible for these studies the patients must have had a histological or cytological diagnosis of pancreatic adenocarcinoma (locally advanced or metastatic). The patients were required to have a Southwest Oncology Group performance status of 0 to 2, a life expectancy of at least 3 months and adequate hematologic, renal and hepatic function. Exclusion criteria were fairly uniform across the three studies and included pregnancy, active malignancy within the preceding 5 years except for adequately treated basal cell, squamous cell skin cancer, or in situ cervical cancer. All patients provided a signed informed consent in accordance with the institutional Human Investigational Committee guidelines prior to enrollment in the study. Serum CA19-9 was obtained at baseline and after each cycle of treatment. For patients with elevated bilirubin at presentation who underwent biliary stenting, CA19-9 level after normalization of serum bilirubin was considered as the baseline.

\section{Statistical methods}

Descriptive statistics were used to summarize patient characteristics and response rates. Exact $90 \%$ confidence limits for response proportions utilized the Casella method $^{7}$ and were computed in StatXact. ${ }^{8}$ Response rates were compared via Fisher's exact test. Responses were determined according to the World Health Organization criteria for the earlier two studies, and by Response Evaluation Criteria in Solid Tumors criteria in the most recent third study. TTP was defined as the time from study registration until computed tomography (CT) documentation of disease progression or death, whichever came first. Patients who were progression free were censored as at the last date of their tumor assessment. OS was defined as the time from study registration until death from any cause, with 
censoring of patients still alive as at the last date that was determined by any method. Standard Kaplan-Meier estimates of the censored TTP and OS distributions were computed. Due to the modest sample sizes, survival statistics (e.g. median, and confidence interval [CI] estimates) were estimated more conservatively using a linear interpolation ${ }^{9}$ among successive event times on the Kaplan-Meier curves. Censored TTP and OS distributions were compared using the log-rank test. ${ }^{9}$

Multivariable Cox proportional hazard ( $\mathrm{PH}$ ) regression models ${ }^{10}$ were used to examine the association of (median-dichotomized) baseline CA19-9 $(<1000 \mathrm{ng} / \mathrm{mL}$ $v s \geq 1000 \mathrm{ng} / \mathrm{mL}$ ) with TTP. The model controlled for the following covariates: stage (locally advanced, metastatic), race (black, white) and age group ( $<56$ years, $56+$ years). The statistical strength of associations between every pair of candidate predictors was examined via Fisher's exact test. If near colinearity was indicated, only one of those covariates was used in the multivariable Cox modeling. That occurred for performance status (PS) and CA19-9 dichotomy, hence we could not adjust for PS as a covariate. The PH assumption was tested by including an interaction (product term) of each independent variable with the natural logarithm of TTP. PH was accepted if that interaction term was not significant $(P>0.05) .{ }^{11}$ There was no evidence of significant non-PH in TTP for any of the predictor variables. First order statistical interactions between baseline CA19-9 dichotomy and each of the three covariates listed above were also examined in the Cox models. Hence, the initial model included five main effects and four interaction terms. One significant interaction term was detected (sex with CA19-9 group), so sex was used as a stratification variable in a subsequent Cox model. The other interaction terms were dropped and a four-variate main effects only, sex-stratified model was refitted. ${ }^{12}$ Goodness of fit of the final Cox model of TTP was evaluated using plots of deviance residuals. ${ }^{11} \mathrm{~A}$ completely analogous Cox modeling investigation was performed for OS and resulted in the same four-variate main effects only, sex-stratified final model.

\section{RESULTS}

Table 1 summarizes the three clinical trials with respect to chemotherapy regimen, accrual time period and population size. There were no statistically significant differences across the three trials in CA19-9 levels, either at baseline or after the first cycle of chemotherapy.
Table 2 Patient characteristics $(N=111)$

\begin{tabular}{lcc}
\hline Characteristics & Number & Percentage \\
\hline Median age (range) & $56(28-81)$ & \\
Sex & & \\
$\quad$ Female & 45 & 41 \\
$\quad$ Male & 66 & 59 \\
Race & & \\
$\quad$ African Americans & 23 & 21 \\
$\quad$ Caucasian & 86 & 77 \\
$\quad$ Other & 2 & 2 \\
Performance status & & \\
$\quad 0$ & 21 & 19 \\
1 & 78 & 70 \\
$\quad 2$ & 12 & 18 \\
Stage & & 82 \\
$\quad$ Locally advanced & 20 & \\
$\quad$ Metastatic & 91 & \\
\hline
\end{tabular}

\section{Patients' characteristics}

Table 2 summarizes the baseline characteristics of 111 patients from these three clinical trials. The median age was 56 years. The median PS was 1 (range $0-2$ ). No significant difference was observed across the three trials with respect to age, sex or PS. Race (in three groups) differed significantly by trial $(P=0.0287)$, but not by race in two groups (African Americans, Caucasian: $P=0.1367)$. Overall $18 \%$ of patients had locally advanced pancreatic cancer. Stage differed significantly by trial $(P=0.0004)$, presumably because the most recent trial was restricted to metastatic patients. To control for any possible residual confounding by stage or race those two covariates were included in the multivariable Cox models of TTP and OS.

For all the patients the overall response rate (complete response + partial response) was $21 \%$ (23 of $111,90 \%$ CI $0.15-0.28)$. Median TTP and OS were 5.6 months (90\% CI 4.4-6.7 months) and 7.8 months (90\% CI 6.88.9 months), respectively.

\section{Relationship between the baseline CA19-9 levels and treatment outcomes}

The baseline CA19-9 measurement was available for 102 patients. It was dichotomized near the median as $<1000$ vs $\geq 1000 \mathrm{ng} / \mathrm{mL}$, as in other studies. ${ }^{13}$ There were 49 patients with baseline CA19-9 $<1000 \mathrm{ng} / \mathrm{mL}$ and 53 patients with CA19-9 $\geq 1000 \mathrm{ng} / \mathrm{mL}$. Responserates did not differ significantly between patients in the lower versus the higher baseline CA19-9 categories (22\% vs $19 \%$, respectively; $P=0.82$ ). 
Figure 1 Kaplan-Meier graph of time to progression (TTP) by category of baseline carbohydrate antigen 19-9 (CA19-9) at levels $(-)<1000$ and (-----) $>1000$. For the 49 patients with baseline CA19-9 < $1000 \mathrm{ng} / \mathrm{mL}$, median TTP was 6.4 months $(90 \%$ CI 5.1-8.2) and their 1-year progressionfree rate was $20 \%(90 \%$ CI 0.09 0.32 ). For the 53 patients with baseline CA19-9 > $1000 \mathrm{ng} / \mathrm{mL}$, the median TTP was 4.2 months (90\% CI 2.6-5.6) and their 1-year progression-free rate was $7 \%(90 \%$ CI $0.00-0.15)$. Logrank test $P=0.0044$. Of the 102 patients with a baseline CA19-9 measurement, 96 had progressed.

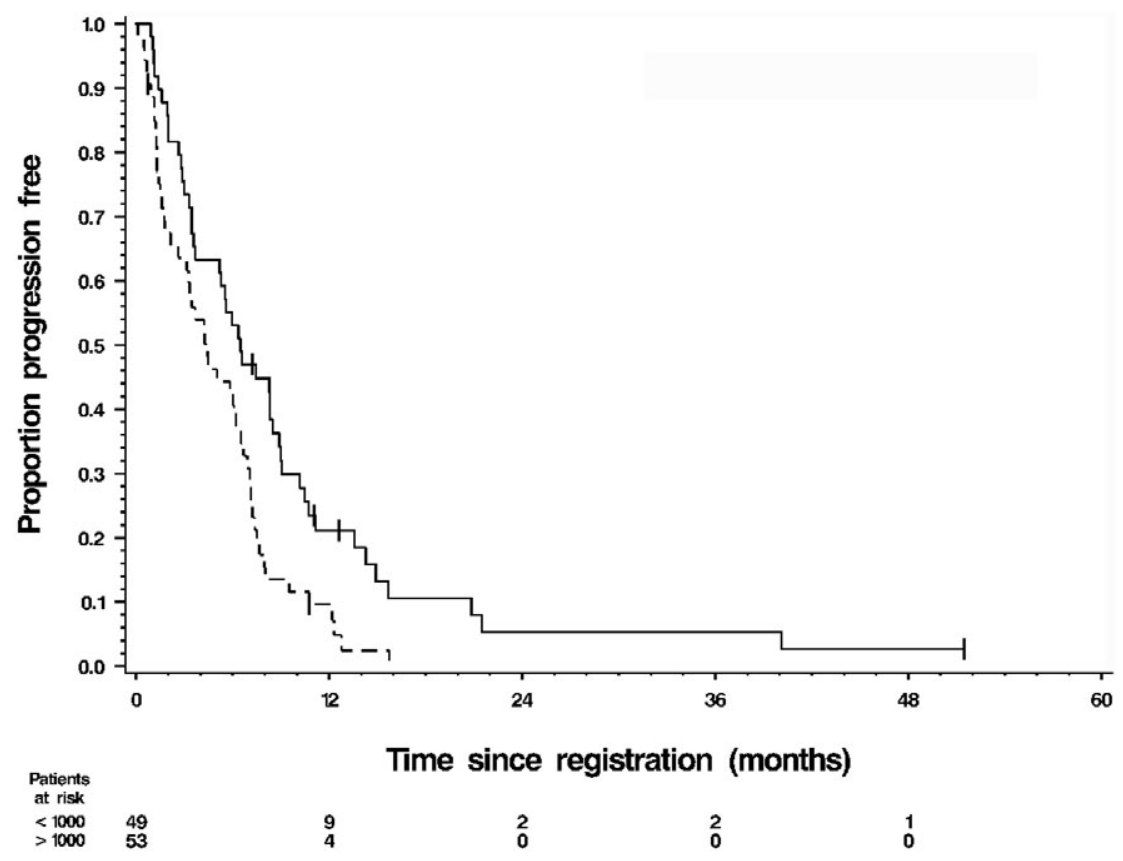

The median TTP for patients in the lower and upper halves of baseline CA19-9 was 6.4 and 4.2 months, respectively (see Fig. 1 ). TTP was statistically significantly different between the two groups $(P=0.0044)$. Median OS for patients in the lower and upper halves of baseline CA19-9 was 9.1 and 6.1 months, respectively (Fig. 2). From a univariate Cox model of TTP, the HR for progression among patients with high (above median) baseline CA19-9 was 1.82 (95\% CI 1.20-2.77, $P=0.0049$ ). This association persisted after adjustment for stage, race and age group in a sex-stratified Cox model of TTP which yielded HR $=1.94$ (95\% CI 1.243.02, $P=0.0035$ ).

OS was statistically significantly different between these two groups $(P=0.0057)$. The 1 -year survival rate was $38 \%$ for patients with CA19-9 $<1000 \mathrm{ng} / \mathrm{mL}$ and $15 \%$ for patients with CA19-9 $\geq 1000 \mathrm{ng} / \mathrm{mL}$. From a univariate Cox model of OS, the HR for death among patients with high (above median) baseline CA19-9 was 1.77 (95\% CI 1.18-2.67, $P=0.0062)$. This association persisted after adjustment for stage, race and age group in a sex-stratified Cox model of OS which yielded $\mathrm{HR}=1.90(95 \%$ CI 1.23-2.94, $P=0.0039)$.

\section{Predictive role of CA19-9 after first cycle of chemotherapy}

Of the 102 patients with available pretreatment CA19-9 levels, the CA19-9 measurement after the first cycle of chemotherapy was available for 68 patients. Of those 68 patients, one patient had disease progression within the first month of enrollment and was excluded from analyses. The effective sample size was 67 for response and TTP and 68 for OS. Patients were divided according to the magnitude of decrease (yes/no) in CA19-9 after the first cycle of chemotherapy using the three cut-off points $\geq 1 \%, \geq 20 \%$ and $\geq 50 \%$. Of the 67 patients, 46 had $\geq 1 \%$ decline, 34 had $\geq 20 \%$ decline and 17 had $\geq 50 \%$ decline.

No significant difference in response rate was observed between those who had a decline in CA19-9 after the first cycle of chemotherapy and those who did not, irrespective of the magnitude of decline (Table 3). The largest difference occurred among patients who had $a \geq 20 \%$ decrease in CA19-9, with 34 patients having a $29 \%$ response rate compared to a $21 \%$ response rate among the 33 patients showing either smaller decreases or actual increases in CA19-9. However, this difference in response rates was not statistically significant. For the 31 stable disease (SD) patients, the median change in CA19-9 was $-18.9 \%$, that is, a decrease. Thus, almost half of the SD patients achieved $\geq 20 \%$ decline in their CA19-9 level.

Patients with a decrease in CA19-9 after the first cycle of chemotherapy had only slightly longer TTP than did patients without such a decrease. Patients who had no decrease in CA19-9 had the worst disease progression 


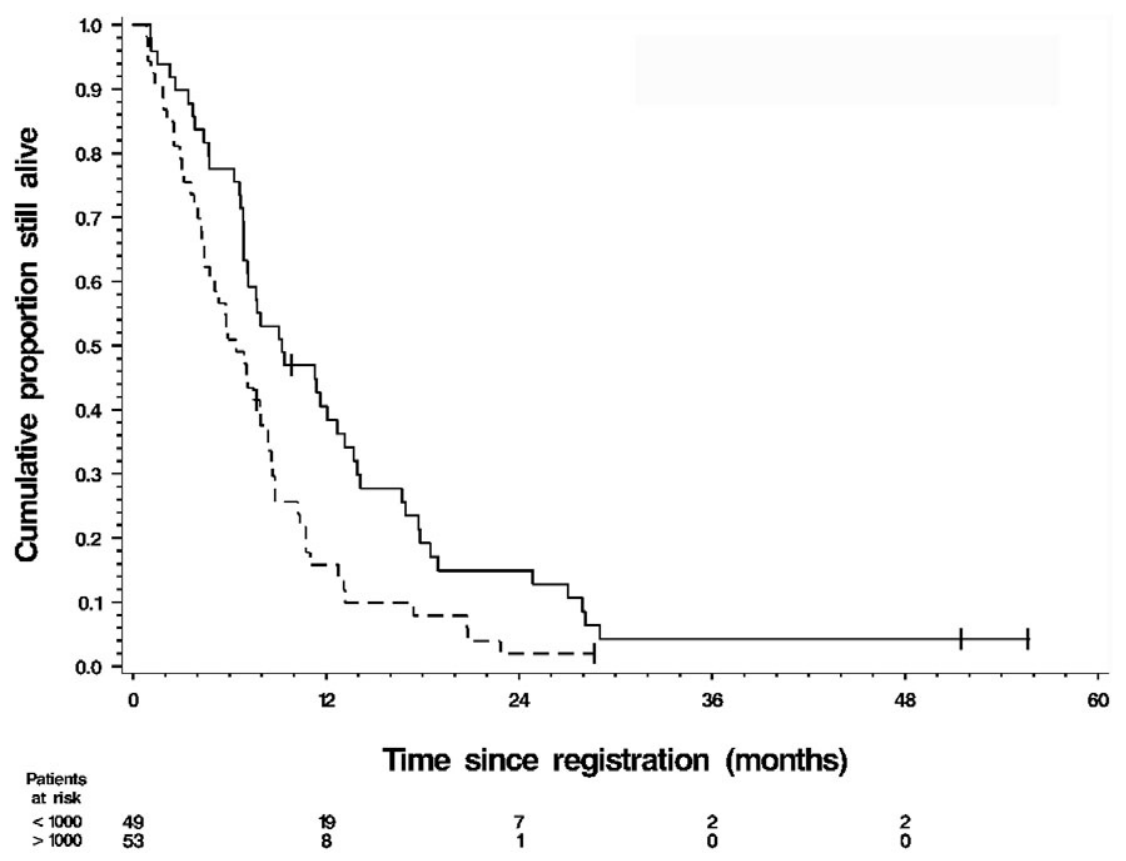

Figure 2 Kaplan-Meier graph of overall survival (OS) by category of baseline carbohydrate antigen 19-9 (CA19-9) at levels $(-)<1000$ and (-----) >1000. For the 49 patients with baseline CA19-9 $<1000 \mathrm{ng} / \mathrm{mL}$ the median OS was 9.1 months $(90 \%$ CI 7.0-11.9) and the 1-year survival rate was $38 \%$ (90\% CI $0.25-0.52)$. For the 53 patients with baseline CA19$9>1000 \mathrm{ng} / \mathrm{mL}$ the median OS was 6.1 months (90\% CI 4.5-7.7) and the 1-year survival rate was $15 \%(90 \% \mathrm{CI}$ 0.05-0.24). Log-rank test $P=0.0057$. Of the 102 patients with a baseline CA19-9 measurement, 97 had died.

Table 3 Comparison of response rate, time to progression, and overall survival by dichotomy of percentage change in carbohydrate antigen 19-9 (CA19-9) after the first cycle of gemcitabine-based chemotherapy

\begin{tabular}{|c|c|c|c|}
\hline Size of decrease in CA19-9 & Yes $^{\dagger}$ & No & $P$-value $\ddagger \$$ \\
\hline$\geq 1 \%$ decrease & $\mathrm{N}=46$ & $\mathrm{~N}=21$ & \\
\hline Response, $n(\%)$ & $12(26 \%)$ & $5(24 \%)$ & 1.00 \\
\hline Median TTP (months) (90\% CI) & $6.3(5.4-7.3)$ & $3.9(1.9-6.9)$ & 0.36 \\
\hline Median OS (months) $(90 \% \mathrm{CI})$ & $8.2(6.9-10.1)$ & $6.5(4.7-8.7)$ & 0.14 \\
\hline$\geq 20 \%$ decrease & $\mathrm{N}=34$ & $\mathrm{~N}=33$ & \\
\hline Response, $n(\%)$ & $10(29 \%)$ & $7(21 \%)$ & 0.58 \\
\hline Median TTP (months) (90\% CI) & $6.5(6.0-7.4)$ & $3.9(3.1-6.9)$ & 0.83 \\
\hline Median OS (months) $(90 \% \mathrm{CI})$ & $8.5(7.0-10.2)$ & $6.7(4.6-8.6)$ & 0.55 \\
\hline$\geq 50 \%$ decrease & $\mathrm{N}=17$ & $\mathrm{~N}=50$ & \\
\hline Response, $n(\%)$ & $5(29 \%)$ & $12(24 \%)$ & 0.75 \\
\hline Median TTP (months) (90\% CI) & $7.1(5.5-7.6)$ & $5.4(3.3-6.4)$ & 0.81 \\
\hline Median OS (months $(90 \% \mathrm{CI})$ & $8.7(7.1-10.3)$ & $7.1(6.6-8.4)$ & 0.73 \\
\hline
\end{tabular}

${ }^{\dagger}$ For OS only, $N=47,35$, and 18 for Yes patients in the three successive dichotomies, respectively; ${ }^{\ddagger}$ From Fisher's exact test for comparison of

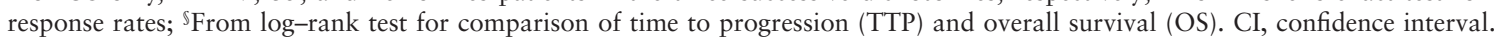

outcome (median TTP 3.9 months, with a lower $90 \%$ confidence limit of 1.9 months).

Overall survival was not significantly different between patients who demonstrated a decline in CA19-9 and those who did not, although it was slightly longer for those who did experience a decline. The best survival outcome was seen among those who had at least a $50 \%$ decrease in CA19-9 (median OS 8.7 months). Patients who had less than $1 \%$ decrease or actual increase had the worst OS (median 6.5 months).
Since no significant univariate association was found between any magnitude of decrease in CA19-9 and any of our three endpoints, no multivariable modeling of these associations was necessary.

\section{DISCUSSION}

The prognosis of patients with locally advanced or metastatic pancreatic cancer remains dismal. The impact of modern therapies including targeted agents remains 
very modest. It may be important to assess response to chemotherapy early in the course of treatment in order to guide treatment decisions and offer prognostic information. The CA19-9 response has emerged as a potential surrogate marker for evaluating the patient's response to chemotherapy. The present study focuses on the association of pretreatment CA19-9 with the outcome for patients with advanced pancreas cancer as well as the relationship between the change in CA19-9 concentrations at the completion of first cycle of therapy prior to radiological evaluation and outcome with respect to response, TTP and survival. The goal of this analysis was to evaluate the predictive role of CA 19-9 for treatment, especially early in the course of therapy.

There was a statistically significant difference in TTP and OS in patients with the lower baseline CA19-9 levels using a cut-off point of $1000 \mathrm{ng} / \mathrm{mL}$. This relationship could not be accounted for by the simultaneous adjustment for stage, race and age group in multivariable Cox models. Saad et al. retrospectively analyzed the pre-treatment CA19-9 levels in 28 patients with advanced pancreatic cancer treated with gemcitabine. They found that patients who had CA19-9 below the median for the entire sample $(1212 \mathrm{U} / \mathrm{mL})$ survived twice as long as those with a CA19-9 above the median (14.9 vs 7.4 months, $P=0.0013)$. In multivariable analysis, baseline CA19-9 level was an independent and stronger predictor of survival than CA19-9 decline on therapy. ${ }^{14}$ Maisey et al. examined the significance of baseline CA19-9 in 154 patients treated with both gemcitabine and 5-fluorouracil or with single agent 5-fluorouracil. OS in patients with a baseline CA19-9 below the median value $\left(958 \mathrm{U} \mathrm{mL}^{-1}\right)$ was significantly longer than OS in patients with levels above the median. The 1-year OS rates were $46 \%$ and $19 \%$, respectively, for patients with a baseline CA19-9 below the median value versus those with a baseline CA19-9 above it. ${ }^{13}$ Our study confirms the findings of a positive association between lower baseline CA19-9 concentration and OS. CA19-9 levels may therefore be used as a stratification factor in randomized clinical trials.

Recent reports have examined the role of CA19-9 response to gemcitabine and the kinetics of CA19-9 as a surrogate of the response to such treatment. Most studies determined the change from baseline at a subsequent point of approximately 8 weeks. This coincided with two cycles of therapy, which is often a time when imaging tests will be performed. Halm et al. examined 43 patients with locally advanced or metastatic pancreatic cancer treated with gemcitabine, and demonstrated that a $>20 \%$ decrease in baseline CA19-9 level after 8 weeks of treatment was associated with a longer median survival than a rise or a $<20 \%$ decrease in the baseline CA19-9 level. ${ }^{15}$ Rocha Lima et al. studied CA19-9 responses in 44 patients with advanced pancreatic cancer treated with gemcitabine and irinotecan. A strong correlation between CA19-9 progression (defined as a greater than 50\% increase in CA19-9 when the lowest on-study level was $200 \mathrm{ng} / \mathrm{mL}$ or lower or a greater than $25 \%$ increase in CA19-9 when the lowest level was higher than $200 \mathrm{ng} / \mathrm{mL}$ ) and TTP $(\mathrm{r}=0.89, P<0.001)$, with CA19-9 progression preceding radiographic progression in most patients. ${ }^{16}$ Ziske et al. studied the CA19-9 response in 46 patients with locally advanced or metastatic pancreatic cancer and found that those with a decrease in CA19-9 levels of $>20 \%$ from the baseline level after 8 weeks of chemotherapy had a significantly longer survival than patients with a rise or decline $<20 \%$. The response of CA19-9 $>20 \%$ during chemotherapy was the only independent predictor of survival in multivariable analyses. In contrast, neither an objective tumor response nor a clinical benefit response showed this level of association. ${ }^{17}$ Stemmler et al. prospectively studied the kinetics of CA19-9 in patients with locally advanced or metastatic pancreatic cancer treated with gemcitabine and cisplatin and found that, independent of the response evaluation by CT imaging, CA19-9 responders (defined as patients achieving $\geq 50 \%$ decrease in CA19-9 serum levels within 2 months after treatment initiation) survived significantly longer than CA19-9 nonresponders. Median survival was 295 days versus 174 days, respectively. ${ }^{18}$ Ko et al. retrospectively evaluated the decline in CA19-9 in 76 patients with advanced pancreatic cancer receiving a fixed dose rate of gemcitabine pooled from three separate studies. They found statistically significant associations between the percentage of CA19-9 decline and both OS and time to treatment failure. Median OS was 12 months for the patients with a $>75 \%$ decline in CA19-9, and only 4.3 months for the patients who did not achieve any decline at all. In their study, the decline in baseline CA19-9 was defined as occurring at any point in time after the initiation of treatment, whether early or late, and is counted as biomarker response, although a significant proportion of patients who responded did so within the first 2 months of therapy. ${ }^{19}$

The early ( $\leq 4$ weeks) determination of response to therapy is beneficial in clinical trials when the prolonged use of a futile experimental drug can be avoided in a patient. In the present study the CA19-9 response after 
the first cycle of chemotherapy was investigated. Patients with $\geq 50 \%$ decline in CA19-9 after the first cycle of chemotherapy had a higher response rate, though it was not statistically significant, than did patients with no decline or with an actual increase. TTP and OS were longer for patients with $\geq 50 \%$ decline after cycle 1 but not statistically significantly so. Therefore, a 50\% decline in CA19-9 after the first cycle ofchemotherapy was not a useful predictor of response, TTP or OS in our study.

There are several possible explanations for the failure to demonstrate the prognostic significance of CA19-9 very early in the course of treatment: (i) the short natural history of locally advanced and metastatic pancreatic cancer may make it difficult to show the impact of a tumor marker that can reliably assess response very early in the course of treatment; (ii) poor treatment alternatives rather than the adequacy of the marker itself; and (iii) the small sample size, as a larger sample size may be required to show such an effect. Even though a change in CA19-9 after the first cycle of chemotherapy was not useful, the pattern of change over time, especially in the face of SD radiographically, may provide valuable information to guide therapy.

Studies on the validation of biomarkers in patients with cancer face several challenges. One limitation to this analysis may be the inclusion of three different trials with different chemotherapeutic regimens. However, all three trials were gemcitabine-based. Another limitation is the influence of biliary obstruction on the concentrations of CA19-9 in serum. This factor was not considered in data analysis. This is especially a problem for using early ( $\leq 4$ weeks) time points to establish predictive biomarkers because of the impact of biliary tract pathology or interventions, or both, that would falsely elevate CA19-9 levels. A strength of this investigation is its prospective nature and the fact that these trials were conducted in only two institutions, which provides uniformity in the treatment and quantification of CA19-9. Furthermore our study used rigorous statistical modeling that revealed a statistically significant relationship of baseline CA19-9 level to TTP and to OS, even after simultaneous adjustment for stage, race and age group.

In conclusion, our study supports the hypothesis that baseline CA19-9 has significant prognostic value in patients with metastatic or locally advanced pancreatic cancer. Its incorporation into the baseline risk stratification should be considered in future therapeutic studies, especially in the randomized setting.

\section{ACKNOWLEDGEMENTS}

This study was partially supported by NIH Cancer Center Support Grant CA-22453.

\section{REFERENCES}

1 Jemal A, Siegel R, Ward E et al. Cancer statistics 2007. CA Cancer J Clin 2006; 56: 106-30.

2 Burris HA III, Moore MM, Andersen J et al. Improvements in survival and clinical benefit with gemcitabine as first-line therapy for patients with advanced panceas cancer: a randomized trial. J Clin Oncol 1997; 15: 2403-13.

3 Rothenberg ML, Abbruzzese JL, Noore M, Portenoy RK, Robertson JM, Wanebo HJ. A rationale for expanding the endpoints for clinical trials in advanced pancreatic carcinoma. Cancer 1996; 78 (Suppl. 3): 627-32.

4 Koprowski H, Steplewski Z, Mitchell K, Herlyn M, Herlyn D, Fuhrer P. Colorectal carcinoma antigens detected by hybridoma antibodies. Somatic Cell Genet 1979; 5: 95771.

5 Tempero MA, Uchida E, Takasaki H, Burnett DA, Steplewski Z, Pour PM. Relationship of carbohydrate antigen 19-9 and Lewis antigens in pancreatic cancer. Cancer Res 1987; 47: 5501-3.

6 Locker GY, Hamilton S, Harris J Jr et al. ASCOO 2006 update of recommendations for the use of tumor markers in gastrointestinal cancer. J Clin Oncol 2006; 24: 1-9.

7 Casella G. Refining binomial confidence intervals. Can J Stat 1986; 14: 113-29.

8 Mehta CPN. StatXact 6:Statistica Software for Exact Nonparametric Inference, User Manual. Cytel Software Corporation, Cambridge, MA 2003.

9 Lee E. Statistica Methods for Survival Data Analysis, 3rd edn. Wiley \& Sons, New York 2003.

10 Cox DR. Regression models and life tables (with discussion). J R Stat Soc Series B 1972; 34: 187-220.

11 Allison PD (ed.). Survival Analysis Using the SAS System: A Practical Guide. SAS Institute, Cary, NC. 1995.

12 Cantor AB. SAS Survival Analysis Techniques for Medical Research, 2nd edn. SAS Institute, Cary, NC 2003.

13 Maisey NR, Norman AR, Hill A, Massey A, Oates J, Cunningham D. CA19-9 as a prognostic factor in inoperable pancreatic cancer: the implication for clinical trials. Br J Cancer 2005; 93: 740-3.

14 Saad ED, Machado M, Wajsbrot D et al. Pretreatment CA19-9 level as a prognostic factor in patients with advanced pancreatic cancer treated with gemcitabine. Int J Gastrointest Cancer 2002; 32: 35-41.

15 Halm U, Schumann T, Schiefke I, Witzigmann H, Mössner J, Keim V. Decrease of CA 19-9 during chemotherapy with gemcitabine predicts survival time in patients with advanced pancreatic cancer. Br J Cancer 2000; 82: 1013-16.

16 Rocha Lima CM, Savarese D, Bruckner H et al. Irinotecan plus gemcitabine induces both radiographic and CA 19-9 
tumor marker responses in patients with previously untreated advanced pancreatic cancer. J Clin Oncol 2002; 20: 1182-91.

17 Ziske C, Schlie C, Gorschlüter M et al. Prognostic value of CA 19-9 levels in patients with inoperable adenocarcinoma of the pancreas treated with gemcitabine. $\mathrm{Br} J$ Cancer 2003; 89: 1413-17).

18 Stemmler J, Stieber P, Szymala AM et al. Are serial CA 19-9 kinetics helpful in predicting survival in patients with advanced or metastatic pancreatic cancer treated with gemcitabine and cisplatin? Onkologie 2003; 26: 4627.

19 Ko AH, Hwang J, Venook AP, Abbruzzese JL, Bergsland EK, Tempero MA. Serum CA19-9 response as a surrogate for clinical outcome in patients receiving fixed-dose rate gemcitabine for advanced pancreatic cancer. $\mathrm{Br}$ J Cancer 2005; 93: 195-9.

20 Philip PA, Zalupski M, Vaitkevicius VK et al. Phase II study of gemcitabine and cisplatin in the treatment of patients with advanced pancreatic carcinoma. Cancer 2001; 92: 569-77.

21 El-Rayes BF, Zalupski M, Shields AF et al. Phase II study of gemcitabine, cisplatin and infusional fluorouracil in advanced pancreatic cancer. J Clin Oncol 2003; 21: 2920-5.

22 El-Rayes BF, Zalupski M, Shields AF et al. A phase II study of celecoxib, gemcitabine, and cisplatin in advanced pancreatic cancer. Invest New Drugs 2005; 23: 583-90. 